\title{
Effect of Ticagrelor versus Clopidogrel on Inflammatory Bio-Marker in Patients with Chronic Stable Angina after Percutaneous Coronary Intervention
}

\author{
Elora Sharmin ${ }^{1 *}$, Jasmine Fauzia Dewann ${ }^{1}$, Harisul Hoque ${ }^{2}$, Ali Ahasan ${ }^{2}$ and Sheikh Foyez Ahmed ${ }^{2}$ \\ ${ }^{1}$ Department of Pharmacology, Bangabandhu Sheikh Mujib Medical University, Bangladesh \\ ${ }^{2}$ Department of Cardiology, Bangabandhu Sheikh Mujib Medical University, Bangladesh
}

Submission: November 07, 2016; Published: December 15, 2016

*Corresponding author: Elora Sharmin, Department of Pharmacology, Bangabandhu Sheikh Mujib Medical University, Bangladesh.

\begin{abstract}
The aim of the study was to compare the effect of Ticagrelor and Clopidogrel on inflammatory marker in patients of chronic stable angina (CSA) following percutaneous coronary intervention (PCI). This prospective observational study included a total of 100 CSA patients. Patients were divided into two groups, the Ticagrelor and the Clopidogrel treated group (each having 50 patients). The laboratory parameters such as -High sensitive C-reactive protein (hs-CRP), bleeding time and clotting time, were measured and then patients of both groups underwent PCI. The same parameters were again assessed at follow up after 4 weeks of intervention. Comparisons of the laboratory parameters were made between two groups at baseline and at follow up and also within group before and after intervention. In the study at baseline characteristics of patients treated with Ticagrelor and Clopidogrel were almost identical in terms of age, sex, diabetes and hypertension. The inflammatory marker hs-CRP was also similar in both groups at baseline. At follow up hs-CRP was significantly reduced from baseline $19.7 \mathrm{mg} / \mathrm{dl}$ to 1.7 $\mathrm{mg} / \mathrm{dl}$ ( $\mathrm{p}$ value- 0.001 ) in ticagrelor group and $18.4 \mathrm{mg} / \mathrm{dl} \mathrm{to} 2 \mathrm{mg} / \mathrm{dl}$ (p value- 0.001) in clopidogrel group. There was no significant change in bleeding time and clotting time in both groups of patients. It is concluded that both Ticagrelor and Clopidogrel are similar in acting on the inflammatory marker, in patients of chronic stable angina.
\end{abstract}

Abbreviations: CSA: Chronic Stable Angina; PCI: Percutaneous Coronary Intervention; hs-CRP: High sensitive C-reactive Protein; DALYs: Disability Adjusted Life Years; MI: Myocardial Infarction; ACS: Acute Coronary Syndrome; ADP: Adenosine Diphosphate; IL6: Interleukin 6; MPO: Myeloperoxidase; sCD40L: Soluble CD40 Ligand

\section{Introduction}

Coronary heart disease is a major global health problem [1]. Low and middle income countries, including South Asian countries, contribute significantly to the global burden of cardiovascular disease. Murray and Lopez (1997) have suggested that by $2020,78 \%$ of all deaths and $86.3 \%$ of all loss of disability adjusted life years (DALYs) will be attributable to this cause [2,3]. Atherosclerotic process including inflammatory process undergoing in the coronary arteries may lead to angina pectoris, myocardial infarction (MI) and if untreated, to death. The damage due to athero-thrombotic inception and oxidative stress in such conditions plays pivotal role in the progression of the disease [4]. High sensitive C-reactive protein (hs-CRP), TNF $\alpha$ and IL-6 are sensitive markers of inflammation [5]. The hs-CRP exerts a direct role in the expression of cell adhesion molecule; this protein thus may be of great prognostic value
[6]. There is a powerful predictive association between raised serum high sensitive C-reactive protein (hs-CRP) values and the outcome of acute coronary syndrome (ACS) [4]. Baseline values of hs-CRP are indicative of metabolic state associated with athero-thrombotic events. The presence of hs-CRP within most athero-thrombotic plaque suggests that it may contribute to the pathogenesis and complication of cardiovascular disease $[2,7,8]$. High sensitive C-reactive protein (hs-CRP) binds to lipoprotein and activates pro-inflammatory complement $[2,7,8]$.

Ticagrelor, a recently introduced ADP-receptor inhibitor, is a member of the thienopyridine class of adenosine diphosphate (ADP) receptor inhibitors which reduces platelet aggregation by reversibly binding to ADP receptors on platelet membrane $[2,7,8]$. Compared to clopidogrel and prasugrel, ticagrelor inhibit adenosine diphosphate (ADP) induced platelet aggregation 
more rapidly and more consistently to a greater extent both in the healthy subjects and in patients with coronary artery disease including those undergoing percutaneous coronary intervention (PCI) [9] and reduce the risk of death either resulting from vascular cause, myocardial infarction (MI) or stroke [10]. In acute coronary syndrome (ACS) inflammatory processes play active role leading to formation of atheroma. So if the antiplatelet drugs could exert anti-inflammatory effect, might be beneficial for the prevention of morbidity and mortality [11]. In patients of ACS following PCI, the chance of thrombotic phenomenon is increased because of inflammatory reaction. In that situation an antiplatelet drug having anti-inflammatory action could be a better option. Therefore the present study has been designed to compare the anti-inflammatory properties of ticagrelor and clopidogrel in CSA patients following PCI.

\section{Materials and Method}

The present prospective observational study was carried out in the Department of Pharmacology, Cardiology and Microbiology, BSMMU, Dhaka from September 2014 to February 2016. Patients who were to suffer from as a case of chronic stable angina and under-going PCI in cardiology department of BSMMU and also fulfilled the inclusion and exclusion criteria were the study population. Patients were divided into two groups, Ticagrelor and Clopidogrel treated groups (each having 50 patients). In laboratory High sensitive C-reactive protein (hs-CRP), bleeding time, clotting time, were measured and then patients of both groups underwent PCI. The same parameters were again assessed at follow up after 4 weeks of intervention. Comparisons of the laboratory parameters were made between two groups at baseline and at follow up and also within group before and after intervention.

\section{High sensitive CRP}

A highly sensitive CRP assay was performed by nephelometry. The assay uses particle-enhanced immunonephelometry to quantitate CRP in serum samples. Polystyrene particles coated with monoclonal antibodies against CRP become agglutinated when mixed with samples containing CRP. The intensity of light scattering due to the agglutination reaction is measured by the nephelometer and is directly related to the CRP concentration. Samples are automatically diluted 20 -fold by the instrument prior to analysis. The assay was standardized against the reference preparation.

\section{Results}

In this study we observed that hs-CRP was higher in CSA patients in baseline. After treatment the hs-CRP was reduced from baseline to follow-up in ticagrelor group $19.7 \mathrm{mg} / \mathrm{dl}$ to $1.7 \mathrm{mg} / \mathrm{dl}$ (p value- 0.001) and in clopidogrel group $18.4 \mathrm{mg} /$ $\mathrm{dl}$ to $2 \mathrm{mg} / \mathrm{dl}$ (p value- 0.001). After 4 weeks of intervention, both groups showed significant reduction of hs-CRP indicating that anti-inflammatory effect takes place in patients of CSA. However, summarizing the findings of the study, it is evident that both the antiplatelet drugs are effective in improving the status inflammatory marker but effect of ticagrelor appeared to be better than clopidogrel (Figures $1 \& 2$ ).

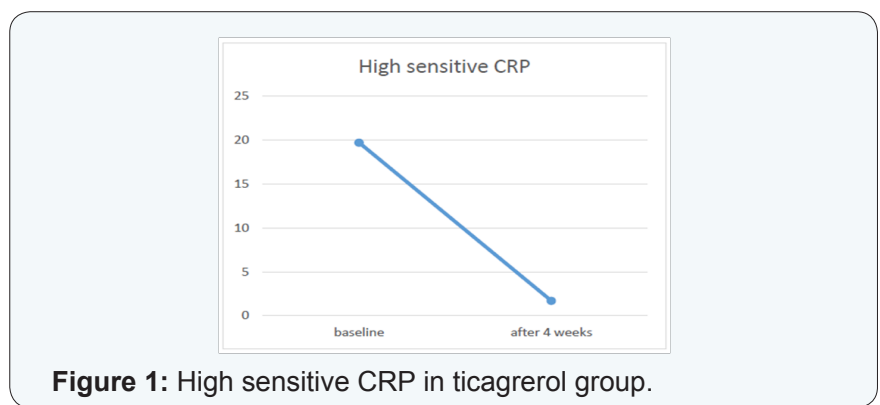

Figure 1: High sensitive CRP in ticagrerol group.

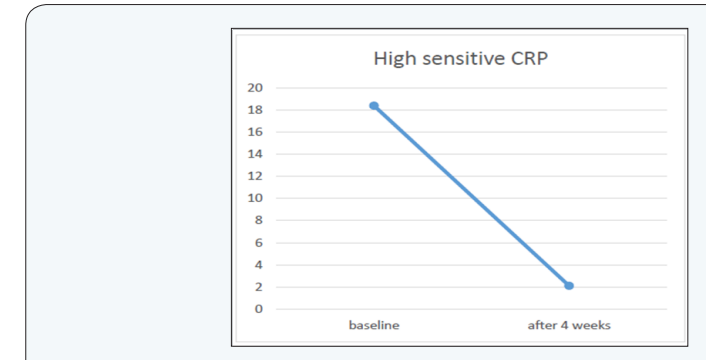

Figure 2: High sensitive CRP in clopidogrel group.

\section{Discussion}

Coronary artery disease is the major cause of mortality and morbidity worldwide [12]. Inflammation is a key etiological factor in the development of atherosclerotic disease and acute coronary syndromes (ACS) [4,13,14]. Many inflammatory biomarkers have been studied as both prognostic indicators and possible intervention targets. Among these are the inflammatory biomarkers interleukin 6 (IL 6), High sensitive C-reactive protein (hs-CRP), myeloperoxidase (MPO), and soluble CD40 ligand ( $\mathrm{SCD} 40 \mathrm{~L})$, which represent pathophysiological steps in the inflammatory process that may contribute to the pathogenesis of ACS [15]. Ticagrelor and Clopidogrel both groups were almost identical in terms of their demographic characteristics (age and sex). The clinical characteristics (diabetes and hypertension) were also identically distributed between groups. The inflammatory marker high sensitive C-reactive protein was almost similar between the study groups. As most of the baseline characteristics were almost similar in distribution between groups, the outcome obtained could be considered due to intervention by drugs.

Inflammation plays a role in the development of atherosclerosis and coronary heart disease [16]. Elevated markers of inflammation, particularly high sensitive CRP are associated with increased risk of cardiovascular events [17-20]. Previously, the clinical benefits of antiplatelet therapy with the P2Y12 receptor inhibitor clopidogrel in ACS patients compared with placebo by reducing the cardiovascular deaths and nonfatal MI or stroke [21]. Treatment with ticagrelor, the first reversibly binding oral P2Y12 receptor inhibitor, results in greater inhibition of platelet aggregation than clopidogrel in patients with stable atherosclerotic disease or ACS $[2,7,8]$. Some studied 
suggest that an anti-inflammatory effect may contribute to the clinical efficacy of P2Y12 inhibitor. After 4 weeks of intervention, both groups showed significant reduction hs-CRP indicating that anti-inflammatory effect takes place in patients of CSA. However, summarizing the findings of the study, it is evident that both the antiplatelet drugs are effective in improving the status inflammatory marker but ticagrelor seems to be better than the clopidogrel.

\section{Conclusion}

From the findings of the study, it appears that both ticagrelor and clopidogrel are effective in improving the status of inflammatory marker resulting from inflammatory process caused by chronic stable angina. But in terms of outcome, ticagrelor could be considered better than clopidogrel.

\section{References}

1. Levi F, Lucchini F, Negri E, La Vecchia C (2002) Trends in mortality from cardiovascular and cerebrovascular disease in Europe and other areas of the world. Heart 88(2): 119-124.

2. Christopher JL Murray, Alan D Lopez (1997) Mortality by cause for eight regions of the world: Global Burden of Disease study. The Lancet 349(9061): 1269-1276.

3. Peter Libby, Paul M Ridker (1999) Novel inflammatory markers of coronary risk. Circulation 100: 1148-1150.

4. Ridker PM (2007) Inflammatory biomarkers and risks of myocardial infarction, stroke, diabetes, and total mortality: implications for longevity. Nutr Rev 65(12 pt 2): S253- S259.

5. Moukarbel GV, Arnaout MS, Alam SE (2001) C-reactive protein is a marker for a complex culprit lesion anatomy in unstable angina. Clin Cardiol 24(7): 506-510.

6. Husted S, Emanuelsson H, Heptinstall S, Sandset PM, Wickens M, et al. (2006) Pharmacodynamics, pharmacokinetics, and safety of the oral reversible P2Y12 antagonist AZD6140 with aspirin in patients with atherosclerosis: a double-blind comparison to clopidogrel with aspirin. Eur Heart J 27(9): 1038-1047.

7. Husted S, van Giezen JJ (2009) Ticagrelor: the first reversibly binding oral P2Y12 receptor antagonist. Cardiovasc Ther 27(4): 259-274.

8. Husted S, Storey RF, Harrington RA, Emanuelsson H, Cannon CP (2010) Changes in Inflammatory Biomarkers in Patients Treated with Ticagrelor or Clopidogrel. Clin Cardiol 33(4): 206-212.
9. Kade Birkeland, David Parra, Robert Rosenstein (2010) Antiplatelet therapy in acute coronary syndromes: focus on ticagrelor. J Blood Med 1:197-219.

10. Wallentin L, Becker RC, Budaj A, Cannon CP, Emanuelsson H, et al. (2009) Ticagrelor versus Clopidogrel in Patients with Acute Coronary Syndromes. N Engl J Med 361(11): 1045-1057.

11. Pepys MB, Williams G (1999) C-reactive protein and amyloidosis from protein to drug. In: Royal College of physicians of London pp. 397-414.

12. Shreesh K Ojha, Mukesh Nandave, Sachin Arora, Rajeev Narang, Amit $\mathrm{K}$ Dinda, et al. (2008) Chronic administration of Tribulus terrestris Linn. Extract improves cardiac function and attenuates myocardial infarction in rats. International Journal of Pharmacology 4(1): 1-10.

13. Lucas AR, Korol R, Pepine CJ (2006) Inflammation in atherosclerosis: some thoughts about acute coronary syndromes. Circulation 113(17): 728-732.

14. Khuseyinova N, Koenig W (2006) Biomarkers of outcome from cardiovascular disease. Curr Opin Crit Care 12(5): 412-419.

15. Armstrong EJ, Morrow DA, Sabatine MS (2006) Inflammatory biomarkers in acute coronary syndromes, part I: introduction and cytokines. Circulation 113(6): 72-75.

16. Lind L (2003) Circulating markers of inflammation and atherosclerosis. Atherosclerosis 169(2): 203-214.

17.Zairis MN, Manousakis SJ, Stefanidis AS, Papadaki OA, Andrikopoulos GK, et al. (2002) C-reactive protein levels and prognosis after STsegment elevation acute myocardial infarction. Am Heart J 144(5): 782- 789 .

18. Yusuf S, Zhao F, Mehta SR, Chrolavicius S, Tognoni G, et al. (2005) Effects of clopidogrel in addition to aspirin in patients with acute coronary syndromes without ST-segment elevation. N Engl J Med 345(7): 494-502.

19. Azar RR, Kassab R, Zoghbi A, Aboujaoudé S, El-Osta H, et al. (2006) Effects of clopidogrel on soluble CD40 ligand and on high-sensitivity $\mathrm{C}$-reactive protein in patients with stable coronary artery disease. Am Heart J 151(2): 521e1-521e4.

20. Yu-guo Chen, Feng Xu, Yun Zhang, Qiu-shang Ji, Yi Sun, et al. (2006) Effect of aspirin plus clopidogrel on inflammatory markers in patients with non-ST-segment elevation acute coronary syndrome. Chin Med J 119(1): 32-36.

21. Woodward M, Lowe GD, Francis LM, Rumley A, Cobbe SM (2004) A randomized comparison of the effects of aspirin and clopidogrel on thrombotic risk factor and C-reactive protein following myocardial infarction: the CADET trial. J Thromb Haemost 2(11): 1934-1940.

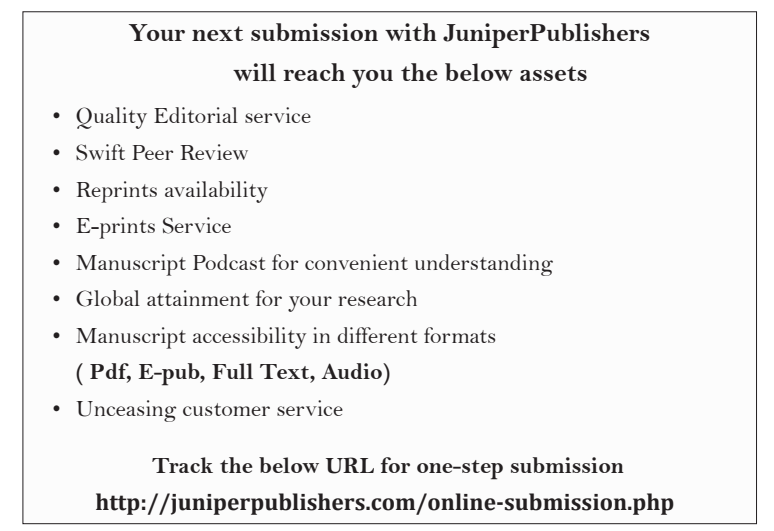

